POS PROCEEDINGS

\title{
Search for the Standard Model Higgs Boson produced in Vector Boson Fusion and decaying into tau pair in CMS with $1 \mathrm{fb}^{-1}$ of luminosity.
}

\author{
Michał Bluj on behalf of the CMS Collaboration*t \\ CNRS/LLR-École Polytechnique, Palaiseau, France \\ E-mail: michal.bluj@cern.ch
}

A prospective analysis is presented on the observability of the Standard Model Higgs boson produced in the Vector Boson Fusion and decaying into tau-tau pair with the tau-tau- $>\ell v v+\tau$-jet $v$ final state. The estimates of the upper limit on the cross section times branching ratio for $1 \mathrm{fb}^{-1}$ for the Higgs boson mass interval $115-145 \mathrm{GeV} / \mathrm{c}^{2}$ are given.

XXth Hadron Collider Physics Symposium

November 16 - 20, 2009

Evian, France

*Speaker.

$\dagger$ On leave from Soltan Institute for Nuclear Studies, Warsaw, Poland 


\section{Introduction}

The Standard Model Higgs boson decay to a tau-tau pair is an important channel in the search of the Higgs boson in the mass range $115-145 \mathrm{GeV} / \mathrm{c}^{2}$. The vector boson fusion (VBF) process, with the second highest cross section at the LHC, provides a characteristic signature of two forward quark jets, which can be used to distinguish the Higgs boson signal from the background processes.

\section{Mass reconstruction}

The full di-tau mass can be reconstructed from two visible taus and $E_{\mathrm{T}}^{\mathrm{miss}}$. For high $p_{\mathrm{T}}$ taus the direction of the neutrinos from its decay is approximately collinear with the visible decay products. Thus, their energies can be retrieved by projecting $E_{\mathrm{T}}^{\text {miss }}$ on visible taus. This collinear approximation provides a satisfactory mass resolution, but a fraction of the events is lost when the approximation results in negative neutrino energy. This may arise from a back-to-back event topology or from a poor reconstruction of $E_{\mathrm{T}}^{\text {miss }}$. Another method, suited for early data-taking, is the di-tau mass reconstruction using only visible decay products. The method is independent on $E_{\mathrm{T}}^{\text {miss }}$ reconstruction, but reconstructed mass is significantly underestimated (Fig. 1).

The dominant uncertainties of the full mass shape are expected to come from the modeling of $E_{\mathrm{T}}^{\text {miss }}$. A method to model the di-tau mass shape from real $Z \rightarrow \mu \mu$ events has been developed. Firstly, muons are removed from the real event and not counted in $E_{\mathrm{T}}^{\text {miss }}$ calculation. Secondly, taus with the real muons kinematics are generated and put into the real $Z \rightarrow \mu \mu$ event to form a di-tau event with fake taus. Finally, the di-tau mass are calculated for this event. Figure 2 shows the reconstructed di-tau mass distribution for the $Z \rightarrow \tau \tau$ events with real and fake taus [1].

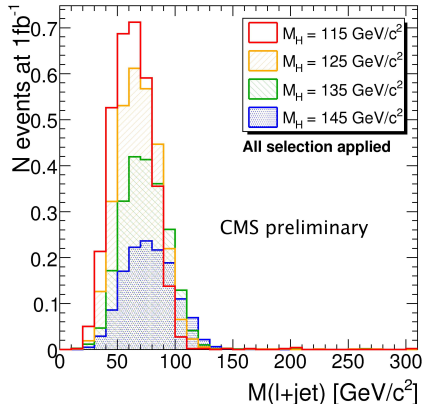

Figure 1: The reconstructed mass distribution using (left) visible decay products and (right) the collinear approximation, for $\mathrm{VBF} \mathrm{H} \rightarrow \tau \tau$ events.

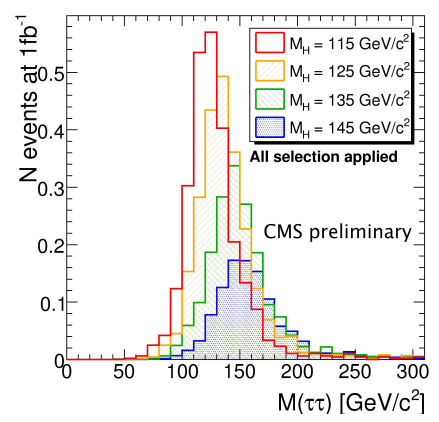

Figure 2: The di-tau mass distribution for $Z \rightarrow \tau \tau \rightarrow \ell v v+\tau_{\text {had }} v$ events with real and fake taus.

\section{Rapidity gap}

A particular signature of VBF events is the rapidity gap between the two quark jets due to the absence of color exchange between the incoming quarks. A selection of the gap allows to reduce significantly background keeping a high efficiency for signal. Two methods of the central rapidity gap selection were studied:

- the Central Jet Veto (CJV) is requirement of non-existence of any jet in $p_{T}>p_{T}^{\min }$ in the rapidity gap, 
- the Track Counting Veto (TCV) is requirement of maximal number of tracks with $p_{T}>p_{T}^{\min }$ in the rapidity gap.

Fig. 3 shows the efficiency of selecting the VBF $H \rightarrow \tau \tau$ signal with respect to the efficiency for the Z+jets background using CJV (left) and TCV (right). For details see in note [1].
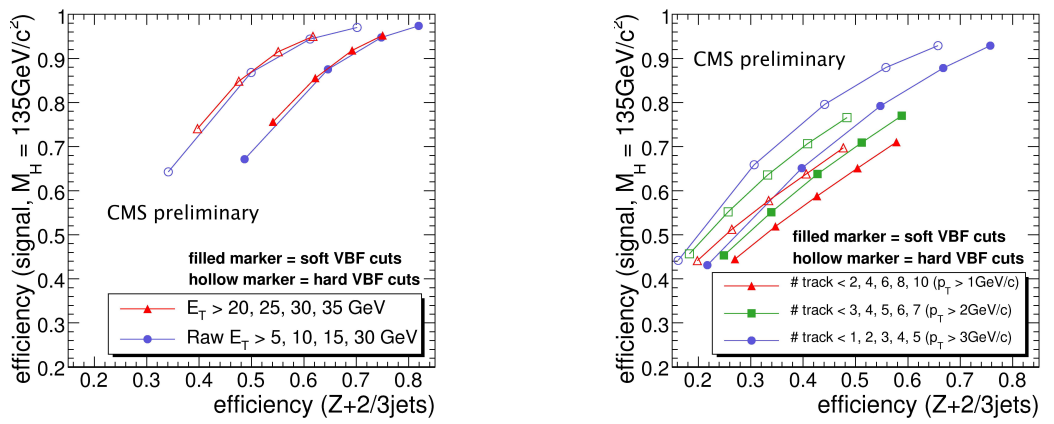

Figure 3: The signal efficiency (VBF H $\left.\rightarrow \tau \tau, M_{H}=135 \mathrm{GeV} / \mathrm{c}^{2}\right)$ vs the background efficiency $(Z+j e t s$, $Z \rightarrow \ell \ell$ ) for CJV (left) and TCV (right).

\section{Results}

The di-tau mass distribution after all selections is shown in Fig. 4. No signal evidence is expected for $1 \mathrm{fb}^{-1}$. The mass distribution for signal and background was used to evaluate the upper limit on the cross section times the branching-ratio in the Higgs mass range 115-145 GeV/c² (Fig. 5). For details see in note [2].

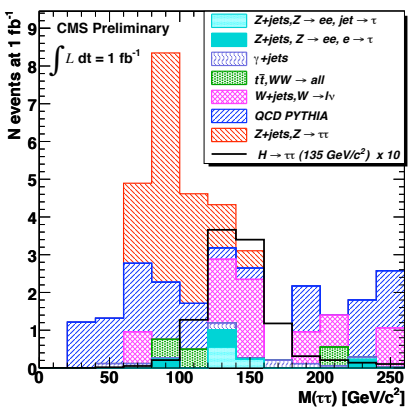

Figure 4: The di-tau mass distributions for the backgrounds expected for $1 \mathrm{fb}^{-1}$ after all selections. The signal mass distribution scaled by factor 10 is also shown for $M_{H}=135 \mathrm{GeV} / c^{2}$.

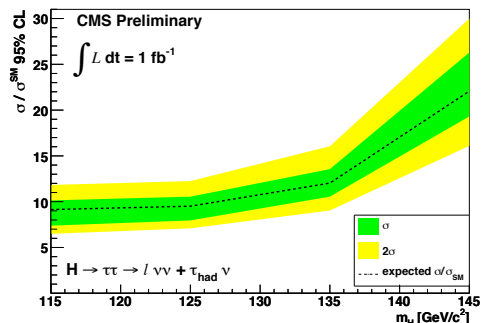

Figure 5: The 95\% CL limit on the cross section times the branching ratio expected for $1 \mathrm{fb}^{-1}$ as a function of the Higgs boson mass. Systematic uncertainties taken into account. The $1 \sigma$ and $2 \sigma$ bands correspond with the statistical uncertainties.

\section{References}

[1] CMS Collaboration, Towards the Search for the Standard Model Higgs boson produced in Vector Boson Fusion and decaying into a $\tau$ pair in CMS with $1 \mathrm{fb}^{-1}: \tau$ identifications studies., CMS PAS HIG-08-001, 2008

[2] CMS Collaboration, Search for the Standard Model Higgs boson produced in Vector Boson Fusion and decaying into a $\tau$ pair in CMS with $1 \mathrm{fb}^{-1}$, CMS PAS HIG-08-008, 2008 